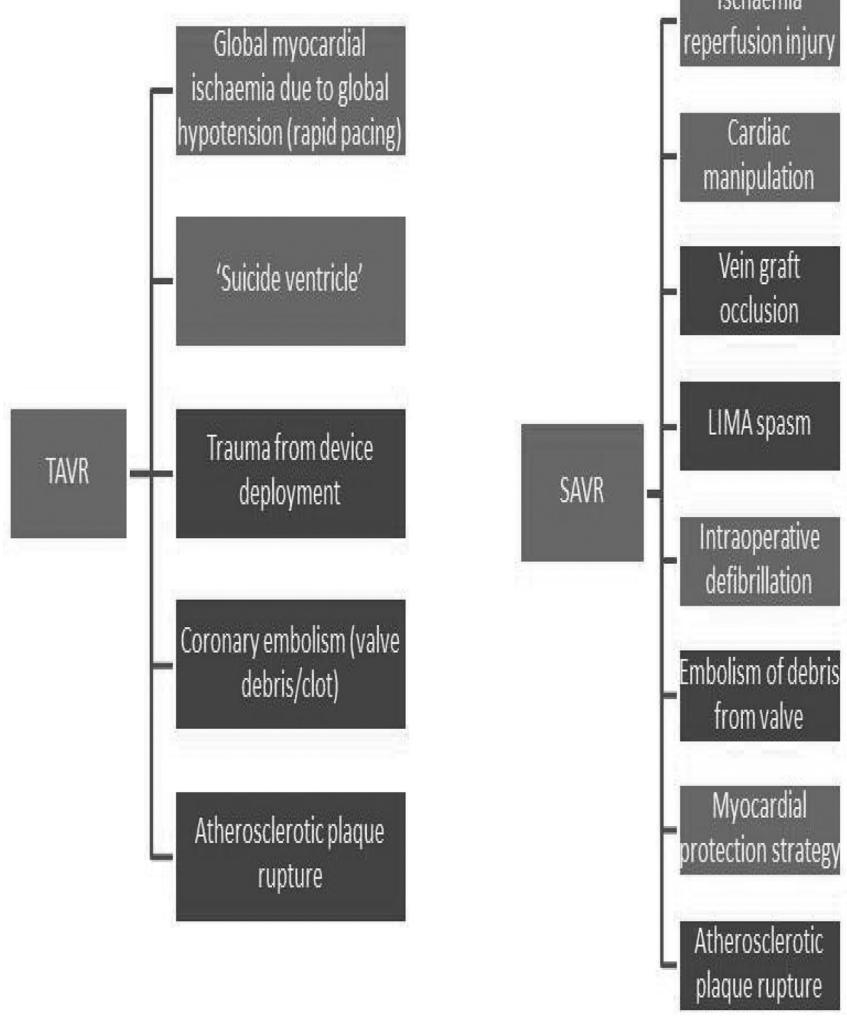

Abstract 38 Figure 1 Mechanisms of Ml

time according to LGE status (New MI $66 \pm 25$ vs. No New MI $84 \pm 42 \mathrm{~min}, \mathrm{p}=0.164)$.

Conclusions MI is an infrequent complication of TAVI but is more common following SAVR. Infarct size is small following both procedures. The low new infarct rate in TAVI, especially in the context of high rates of non-revascularised CAD, is reassuring and strengthens the notion that coronary revascularization prior to TAVI may be unnecessary.

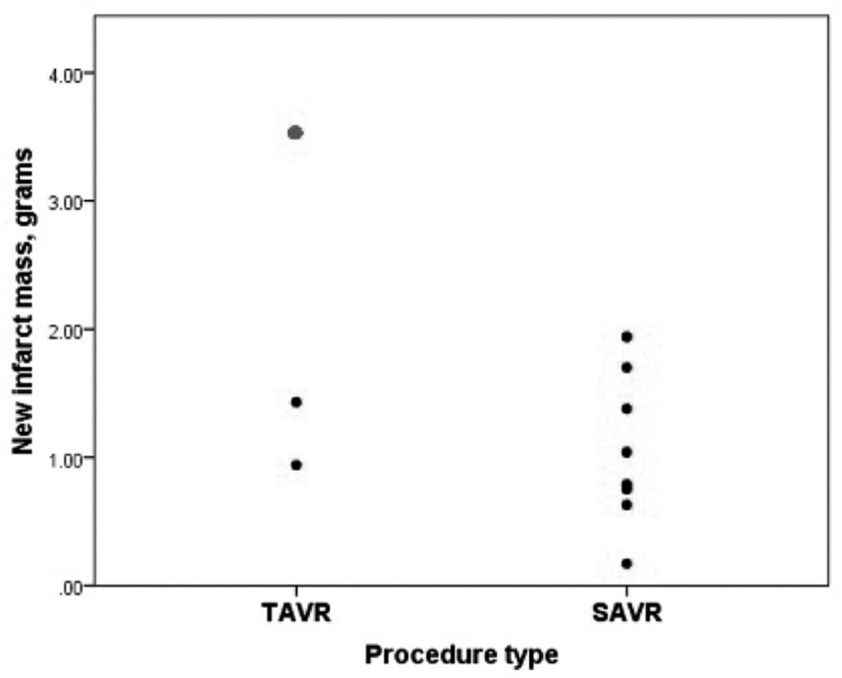

\section{THE IMPACT OF NEW LEFT BUNDLE BRANCH BLOCK FOLLOWING TRANS-CATHETER AORTIC VALVE IMPANTATION. IS THERE A TAVI LBBB-INDUCED CARDIOMYOPATHY? INSIGHTS FROM CARDIOVASCULAR MAGNETIC RESONANCE IMAGING}

${ }^{1}$ Laura E Dobson*, ${ }^{1}$ Tarique A Musa, ${ }^{1}$ Uddin Akhlaque, ${ }^{1}$ Timothy A Fairbairn, ${ }^{2}$ Owen J Bebb, 'Peter P Swoboda, 'Philip Haaf, 'James RJ Foley, ${ }^{1}$ Pankaj Garg, ${ }^{1}$ Graham J Fent, ${ }^{2}$ Christopher J Malkin, 'Daniel J Blackman, 'Sven Plein, 'John P Greenwood. 'University of Leeds; ${ }^{2}$ Leeds Teaching Hospitals NHS Trust; * Presenting Author

\subsection{6/heartjnl-2016-309890.39}

Introduction Left bundle branch block (LBBB) is common following trans-catheter aortic valve implantation (TAVI) and has been linked to increased mortality, although whether this is due to the development of a TAVI-induced LBBB cardiomyopathy is unclear.

Methods 48 patients undergoing TAVI for severe aortic stenosis were evaluated. 24 patients with TAVI-induced LBBB (LBBB-T) were matched with 24 patients with a narrow postprocedure QRS (nQRS). Patients underwent comprehensive Cardiovascular Magnetic Resonance (CMR) imaging prior to and $6 \mathrm{~m}$ post-TAVI. Measured cardiac reverse remodelling parameters included left ventricular ejection fraction (LVEF), global longitudinal strain (GLS) and left sided chamber size. Inter and intraventricular dyssynchrony was determined using time to peak radial strain derived from CMR Feature Tracking.

Results In the nQRS group there was no change in QRSd (93 \pm 17 to $96 \pm 11 \mathrm{~ms}, \mathrm{p}=0.098$ ). In the LBBB-T group, QRSd increased by a mean of $55 \mathrm{~ms}$ from $96 \pm 14$ to 151 $\pm 12(\mathrm{p}=<0.001)$. There was a significant difference in change in LVEF and GLS according to post-procedure QRS duration (LVEF: nQRS $4.6 \pm 7.8$ vs LBBB-T $-2.1 \pm 6.9 \%, p$ $=0.002$; GLS: nQRS $2.1 \pm 3.6$ vs. LBBB-T $-0.2 \pm 3.2 \%, p$ $=0.024$ ) (Figure 1). Those in the $\mathrm{nQRS}$ group had a significant improvement in $\operatorname{LVEF}(54.1 \pm 11.5$ to $58.7 \pm 9.0 \%, \mathrm{p}$ $=0.010)$ and GLS $(15.6 \pm 3.9$ to $17.7 \pm 2.7, \mathrm{p}=0.010)$ at $6 \mathrm{~m}$ follow up. There was a trend towards a reduction in LVEF in the LBBB-T group (56.6 \pm 10.5 to $54.4 \pm 9.3 \%$, p $=0.092$ ). The change in LVEF was driven by a reduction in indexed end-systolic volume in the nQRS group not seen in the LBBB-T group (nQRS $-7.9 \pm 14.0$ vs. LBBB-T $-0.6 \pm$

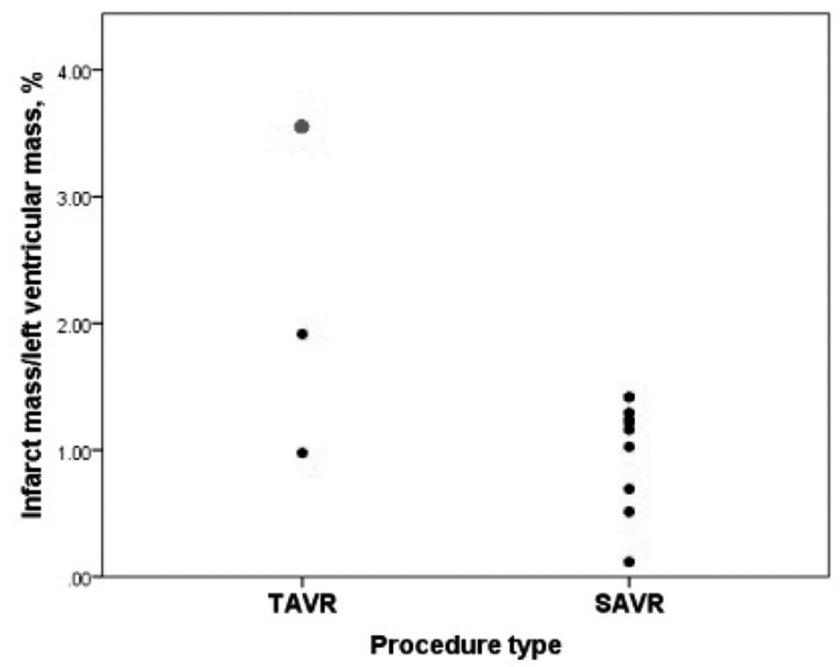

Abstract 38 Figure 2 Graphs of new infarct size 

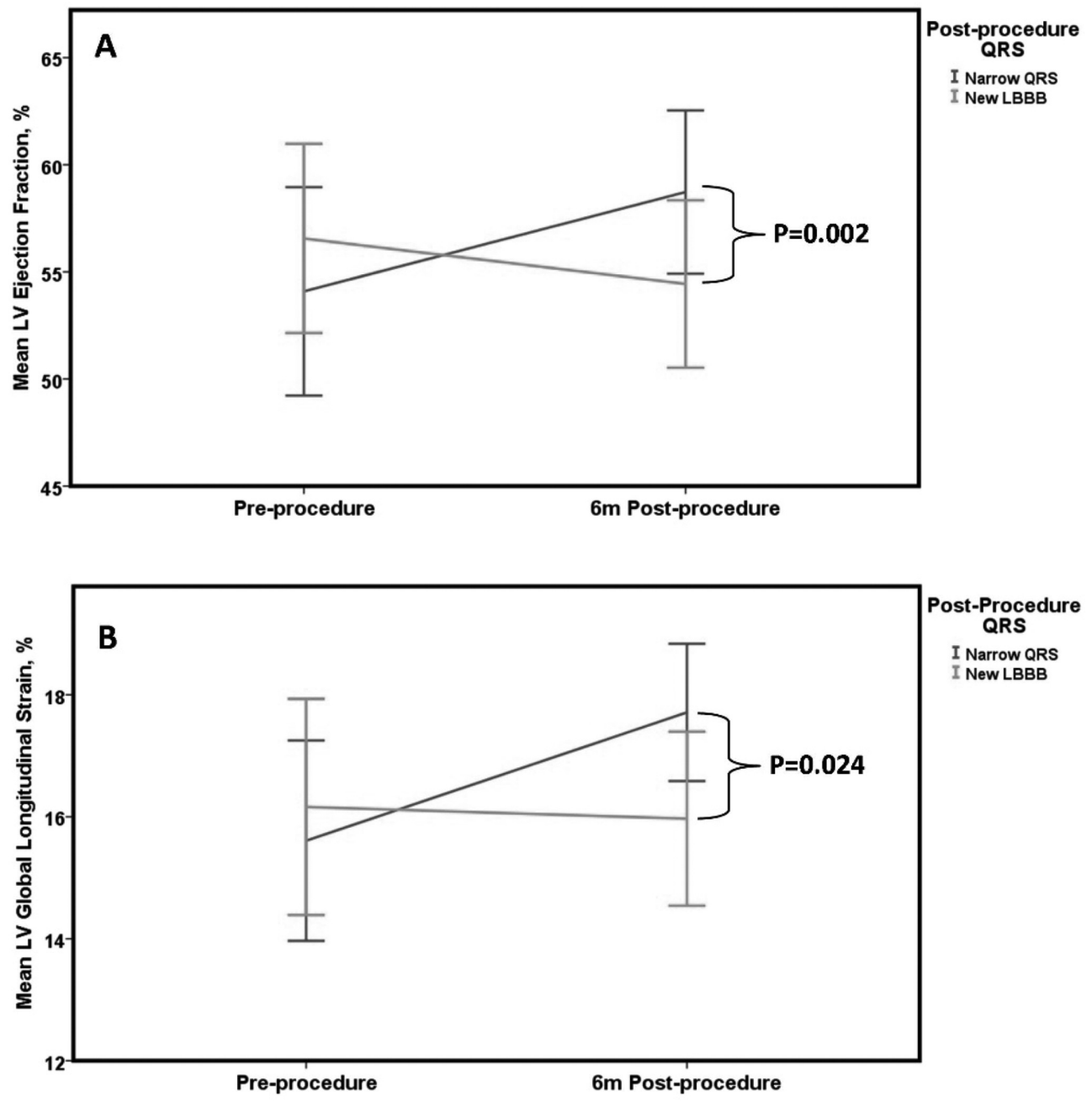

Abstract 39 Figure 1 Change in LVEF \& GLS according to post-TAVI QRS

$\left.10.2 \mathrm{ml} / \mathrm{m}^{2}, \mathrm{p}=0.02\right)$. Further CMR characteristics can be seen in Table 1 . Those with LBBB-T exhibited significant interventricular dyssynchrony $6 \mathrm{~m}$ follow up compared with the nQRS population (LBBB-T $130 \pm 73 \mathrm{~ms}$ vs nQRS $23 \pm 86$ $\mathrm{ms}, \mathrm{p}=<0.001)$. Intraventricular dyssynchrony was also demonstrated in the LBBB-T at $6 \mathrm{~m} ; 118 \pm 103 \mathrm{~ms}$ compared with $13 \pm 106 \mathrm{~ms}(\mathrm{p}=0.001)$ in the nQRS group. There was a significant correlation between post procedure QRS and interventricular and intraventricular dyssynchrony $(\mathrm{r}=0.57$, $\mathrm{p}=<0.001$ and $\mathrm{r}=0.49, \mathrm{p}=<0.001$ respectively). Neither group experienced any change in right ventricular longitudinal function (nQRS $21.7 \pm 7.0$ to $21.5 \pm 6.2 \mathrm{~mm}, \mathrm{p}=0.817$ LBBB-T $18.9 \pm 5.8$ to

$18.6 \pm 5.8 \mathrm{~mm}, \mathrm{p}=0.773)$. Post-procedure aortic regurgitant fraction was similar between groups (nQRS $5.4 \pm 5.7$ vs LBBB-T $5.5 \pm 3.3 \%, \mathrm{p}=0.948$ ). There was an inverse correlation between QRS duration and change in LVEF $(r=-0.46, p$ $=0.001)$ and QRS duration and change in LV GLS $(r=-0.37$, $\mathrm{p}=0.010)$.

Conclusion LBBB-T is associated with less favourable cardiac reverse remodelling at medium term follow up. In view of this, every effort should be made to prevent TAVI-induced LBBB, especially as TAVI is extended to a younger, lower risk population.

\section{QUANTIFICATION OF AORTIC REGURGITATION FOLLOWING TRANSCATHETER AORTIC VALVE IMPLANTATION (TAVI): A CMR STUDY OF TWO PROSTHESIS DESIGNS}

${ }^{1}$ Tarique Al Musa*, ${ }^{1}$ Akhlaque Uddin, 'Laura E Dobson, ${ }^{1}$ Peter P Swoboda, ${ }^{1}$ Pankaj Garg, ${ }^{1}$ Graham J Fent, ${ }^{1}$ James RJ Foley, ${ }^{2}$ Christopher J Malkin, ${ }^{1}$ Sven Plein, ${ }^{2}$ Daniel J Blackman, ${ }^{1}$ John P Greenwood. 'University of Leeds; ' Leeds Teaching Hospitals; *Presenting Author

\subsection{6/heartjnl-2016-309890.40}

Background Transcatheter aortic valve implantation is an established therapeutic option for patients with severe symptomatic aortic stenosis unsuitable for surgery. CMR has a lower intraobserver and inter-observer variability than echocardiography in the assessment of aortic regurgitation (AR), offering full volumetric quantitation independent of the number or eccentricity of regurgitant jets. Moderate AR following TAVI is an independent predictor of mortality in the postoperative period and has prompted development of novel bioprostheses designed to facilitate precise deployment and minimise paravalvular regurgitation (Figure 1).

Aim To compare the degree of early post-procedure aortic regurgitation following implantation of the Medtronic CoreValve and Boston Scientific Lotus Valve systems in patients treated for severe symptomatic aortic stenosis.

Methods All patients underwent an identical 1.5T CMR scan (Intera, Phillips Healthcare, Best, Netherlands) post-procedure 